\title{
Band-gap profiling by laser writing of hydrogen-containing III-N-Vs
}

\author{
N. Balakrishnan, ${ }^{1}$ G. Pettinari, ${ }^{1}$ O. Makarovsky, ${ }^{1}$ L. Turyanska,${ }^{1}$ M. W. Fay, ${ }^{2}$ M. De Luca,${ }^{3}$ A. Polimeni, ${ }^{3}$ M. Capizzi ${ }^{3}$ \\ F. Martelli, ${ }^{4}$ S. Rubini, ${ }^{4}$ and A. Patanè ${ }^{1, *}$ \\ ${ }^{1}$ School of Physics and Astronomy, The University of Nottingham, Nottingham NG7 2RD, United Kingdom \\ ${ }^{2}$ Nottingham Nanotechnology and Nanoscience Centre, The University of Nottingham, Nottingham NG7 2RD, United Kingdom \\ ${ }^{3}$ Dipartimento di Fisica, Sapienza Università di Roma, Piazzale A. Moro 2, 00185 Roma, Italy \\ ${ }^{4}$ TASC-IOM-CNR, Area Science Park, S.S. 14, Km. 163.5, 34149 Trieste, Italy
}

(Received 6 August 2012; published 10 October 2012)

\begin{abstract}
We show that the dissociation of the N-H complex in hydrogenated III-N-Vs can be laser activated at temperatures that are significantly smaller than those $\left(>200^{\circ} \mathrm{C}\right)$ required for thermal dissociation due to a resonant photon absorption by the $\mathrm{N}-\mathrm{H}$ complex. This phenomenon provides a mechanism for profiling the band-gap energy in the growth plane of the III-N-Vs with submicron spatial resolution and high energy accuracy; the profiles are erasable and the alloys can be rehydrogenated making any nanoscale in-plane band-gap profile rewritable.
\end{abstract}

DOI: 10.1103/PhysRevB.86.155307

PACS number(s): 71.55.Eq, 73.21.Fg, 78.20.-e, 78.55.Cr

\section{INTRODUCTION}

Research on the effects of hydrogen on the electronic properties of semiconductor materials and nanostructures is an interesting growing field in condensed matter physics. ${ }^{1-10}$ Hydrogen atoms are highly mobile and reactive elements that bind to several atomic species; they can passivate both deep and shallow crystal defects and impurities in semiconductors ${ }^{3}$ or act as dopants in narrow-band-gap semiconductors. ${ }^{3,6}$ The versatility of hydrogen and its properties are successfully exploited in various applications: hydrogen passivation of defects in $\mathrm{Si} / \mathrm{SiO}_{2}$ is essential for reliable operation of complementary metal-oxide semiconductor technologies; ${ }^{4}$ hydrogen binds to carbon in graphene to transform this highly conductive semimetal into an insulator; ${ }^{5}$ and hydrogenation combined with lithography and masking of an optically active dilute nitride III-N-V alloy is used to form ordered arrays of zero-dimensional nanostructures. ${ }^{7}$ The combination of hydrogenation and laser excitation of a semiconductor can also provide a successful tool for tuning electronic properties. In Ref. 11, we described how a focused laser beam can be used to dissociate $\mathrm{N}-\mathrm{H}$ complexes in hydrogenated $\mathrm{Ga}(\mathrm{AsN})$, thus modifying locally the electronic activity of the $\mathrm{N}$ atoms and the band-gap energy. This recent finding has raised questions of fundamental interest, i.e., whether the physical process that drives the laser-induced modification of the $\mathrm{N}-\mathrm{H}$ bonds in the III-V host crystal is a "thermal" or a "photonic" mechanism and whether this can be used to learn about the physics of $\mathrm{H}$-related defects in III-N-Vs; also, while this laser-assisted band-gap engineering technique was successfully applied to $\mathrm{Ga}(\mathrm{AsN})$, its applicability to other technologically important III-N-V alloys ${ }^{12-15}$ has not yet been examined. These issues are not only of broad fundamental interest in the context of our understanding of the electronic properties of this class of alloys and H-related defects, but also relevant to the development of a versatile and reliable top-down nanofabrication approach and exploitation of III-N-Vs in future nanotechnologies.

In this work, we investigate the laser-induced modification of the $\mathrm{N}-\mathrm{H}$ bonds by microphotoluminescence $(\mu \mathrm{PL})$ spectroscopy and mapping, and secondary electron (SE) imaging. We show that the laser-assisted dissociation of the $\mathrm{N}-\mathrm{H}$ complex in hydrogenated $\mathrm{Ga}(\mathrm{AsN})$ is enhanced at characteristic laser photon energies $h v \sim 1.76 \mathrm{eV}$ that are larger than the band-gap energy of GaAs $(h v=1.43 \mathrm{eV}$ at $T=300 \mathrm{~K})$ and of $\mathrm{Ga}(\mathrm{AsN})(h v=1.30 \mathrm{eV}$ at $T=300 \mathrm{~K}$ for $\mathrm{N}=0.9 \%$ ). We explain this phenomenon in terms of a resonant photon absorption by the $\mathrm{N}-\mathrm{H}$ complex assisted by the photogenerated carriers. This is a local process that can be activated at temperatures that are significantly smaller than those $\left(>200^{\circ} \mathrm{C}\right)$ required for thermal dissociation and provides a means of patterning with submicron spatial resolution in-plane band-gap profiles of different shapes. Nanoscale light emitting spots in the visible and near infrared regions are laser activated in all the III-N-V alloys [i.e., Ga(AsN), (InGa)(AsN), and $\mathrm{Ga}(\mathrm{PN})]$ investigated in this work. Since this direct laser writing technique does not require lithography and etching, it offers a promising route to cost-effective, large-volume and fast nanofabrication methods. The results are therefore of general interest and relevant to our current understanding of the physics and applications of $\mathrm{N}-\mathrm{H}$ defects in III-Vs.

\section{SAMPLES AND TECHNIQUES}

Our work focuses on a $\mathrm{Ga}(\mathrm{AsN}) / \mathrm{GaAs}$ quantum well (QW) sample grown by molecular beam epitaxy (MBE) on a (100)-oriented GaAs substrate with the following layer composition, in order of growth: an undoped GaAs buffer layer grown at $600{ }^{\circ} \mathrm{C}$, a $6 \mathrm{~nm} \mathrm{Ga}(\mathrm{AsN})([\mathrm{N}]=0.9 \%)$ quantum well, and a $30 \mathrm{~nm}$ undoped GaAs cap layer, both grown at $500^{\circ} \mathrm{C}$. The $\mathrm{N}$ concentration was determined by high-resolution $\mathrm{x}$-ray diffraction measurements and further supported by photoluminescence (PL) studies showing that the $\mathrm{Ga}(\mathrm{AsN})$ QW PL emission is redshifted by $0.13 \mathrm{eV}$ relative to the $\mathrm{GaAs}$ PL emission at $T=300 \mathrm{~K}$. This sample was hydrogenated by ion-beam irradiation using a Kaufman source. Postgrowth hydrogenation was performed at $T=200^{\circ} \mathrm{C}$ with an ion-beam energy of $100 \mathrm{eV}$ and impinging $\mathrm{H}$ doses of $4 \times 10^{16}$ ions $/ \mathrm{cm}^{2}$ (sample H1) and $6 \times 10^{16}$ ions $/ \mathrm{cm}^{2}$ (sample H2). The areal density of $\mathrm{H}$ atoms in the samples amounts to about $2 \%$ of the nominal doses quoted above, as derived from secondary 
ion mass spectrometry ${ }^{16}$ and nuclear reaction analysis ${ }^{17}$ on $\mathrm{Ga}(\mathrm{AsN})$ samples similar to those studied in this work. To test the applicability of our laser writing technique to different III-N-Vs, an (InGa)(AsN)/GaAs QW, a Ga(PN) alloy, and a $\mathrm{Ga}(\mathrm{PN}) / \mathrm{GaP}$ multi-QW (MQW) were also examined. The $(\mathrm{InGa})(\mathrm{AsN}) / \mathrm{GaAs} \mathrm{QW}([\mathrm{In}]=32 \%$ and $[\mathrm{N}]=2.7 \%)$ was grown by $\mathrm{MBE}$ on a (100)-oriented GaAs substrate. It has a $6.8 \mathrm{~nm}$ well and a $100 \mathrm{~nm}$ GaAs cap layer. The $\mathrm{Ga}(\mathrm{PN})$ alloy and $\mathrm{Ga}(\mathrm{PN}) / \mathrm{GaP} \mathrm{MQW}$ structures were grown by low pressure (50 mbar) MOVPE using a horizontal reactor system on a $\mathrm{GaP}$ substrate. The $\mathrm{Ga}(\mathrm{PN})$ alloy has $[\mathrm{N}]=0.15 \%$ and thickness of $500 \mathrm{~nm}$. The MQW consists of five QWs. Each QW has a well width of $10 \mathrm{~nm}$, a GaP barrier thickness of $70 \mathrm{~nm}$, and $[\mathrm{N}]=1.1 \%$. These samples were hydrogenated at $T=300^{\circ} \mathrm{C}$ with an ion-beam energy of $100 \mathrm{eV}$ and impinging $\mathrm{H}$ doses of $5 \times 10^{17}$ ions $/ \mathrm{cm}^{2}, 7 \times 10^{18}$ ions $/ \mathrm{cm}^{2}$, and $8 \times 10^{18}$ ions $/ \mathrm{cm}^{2}$ in the (InGa)(AsN) QW, Ga(PN), and $\mathrm{Ga}(\mathrm{PN}) \mathrm{MQWs}$, respectively.

The experimental setup for $\mu \mathrm{PL}$ measurements comprises an $X Y$ linear positioning stage, an optical confocal microscope equipped with a nanofocusing system, a spectrometer with a $150 \mathrm{~g} / \mathrm{mm}$ grating equipped with a CCD and a liquid-nitrogencooled (InGa)As array photodetector. For the PL experiment, we used either an He-Ne laser with $\lambda=633 \mathrm{~nm}(h v=1.96 \mathrm{eV})$ or a frequency-doubled $\mathrm{Nd}: \mathrm{YVO}_{4}$ laser with $\lambda=532 \mathrm{~nm}$ $(h v=2.33 \mathrm{eV})$. The laser beam was focused to a diameter $d<1 \mu \mathrm{m}$ using a $\times 100$ objective and the $\mu$ PL spectra were measured at low power $(P \leqslant 1 \mathrm{~mW})$ at several points by moving the sample with respect to the laser beam in steps of $0.1 \mu \mathrm{m}$. For the laser exposure experiments, we used an Ar laser $(\lambda=515 \mathrm{~nm} ; h v=2.41 \mathrm{eV})$, a Ti:sapphire laser $(\lambda=695-830 \mathrm{~nm} ; h v=1.49-1.78 \mathrm{eV})$, a He-Ne laser $(\lambda=633$ $\mathrm{nm} ; h v=1.96 \mathrm{eV})$, or a frequency-doubled $\mathrm{Nd}: \mathrm{YVO}_{4}$ laser $(\lambda=532 \mathrm{~nm} ; h v=2.33 \mathrm{eV})$, laser annealing powers $P_{a}$ up to $120 \mathrm{~mW}$, and exposure times $t_{a}$ up to $180 \mathrm{~s}$. Images of the sample topography were acquired by atomic force microscopy (AFM) and by scanning electron microscopy (SEM). For the SE imaging, an FEI Quanta 200 3D SEM equipped with an Everhart Thornley detector was used. This was operated at $30 \mathrm{kV}$ for topographical sensitivity and at $1 \mathrm{kV}$ (with a nominal current of $0.11 \mathrm{nA}$ ) to obtain dopant contrast sensitivity. Samples were plasma cleaned in a Fischione Model 1020 plasma cleaner immediately prior to analysis, and images were acquired from single pass scans at $30 \mu$ s dwell to reduce surface contamination contributions.

\section{RESULTS AND DISCUSSION}

Figure 1(a) shows the room temperature $(T=300 \mathrm{~K}) \mu \mathrm{PL}$ spectra and $\mu \mathrm{PL}$ map of one of the hydrogenated $\mathrm{Ga}(\mathrm{AsN})$ samples (H2) after exposure for a time $t_{a}=15 \mathrm{~s}$ to a focused laser beam with diameter $d<1 \mu \mathrm{m}, \lambda=515 \mathrm{~nm}$ $\left(h v=2.41 \mathrm{eV}\right.$ ), and $P_{a}=10 \mathrm{~mW}$. The $\mu \mathrm{PL}$ spectra were acquired at low laser power within and around the laserannealed spot. It can be seen that the QW PL emission is peaked at lower energies in proximity of the spot center [see the $\mu \mathrm{PL}$ spectra at positions A, B, C, and D in Fig. 1(a)]. The $\mu \mathrm{PL}$ map in the spectral range of the QW emission shows a light emitting region with an approximately Gaussian form of its intensity and a full width at half maximum
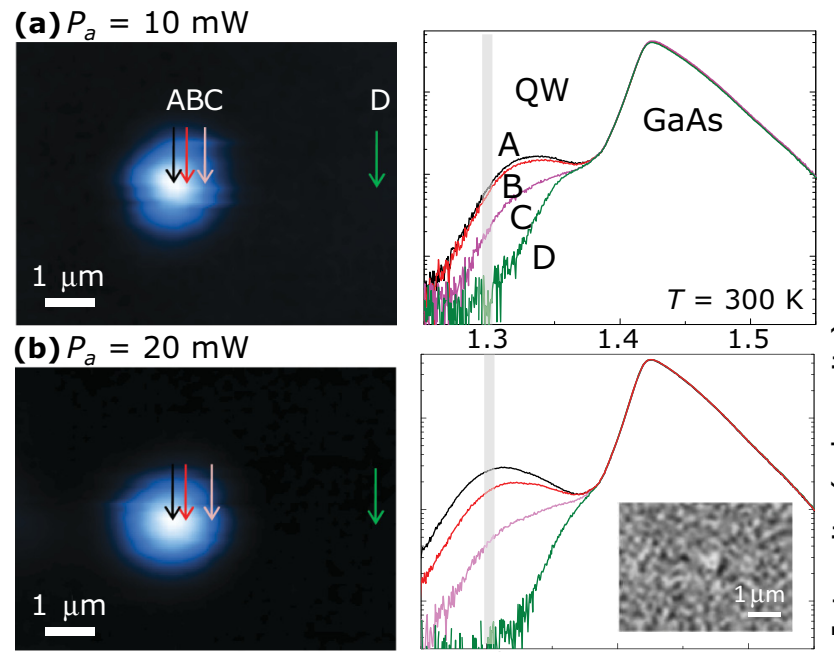

(c) $P_{a}=40 \mathrm{~mW}$
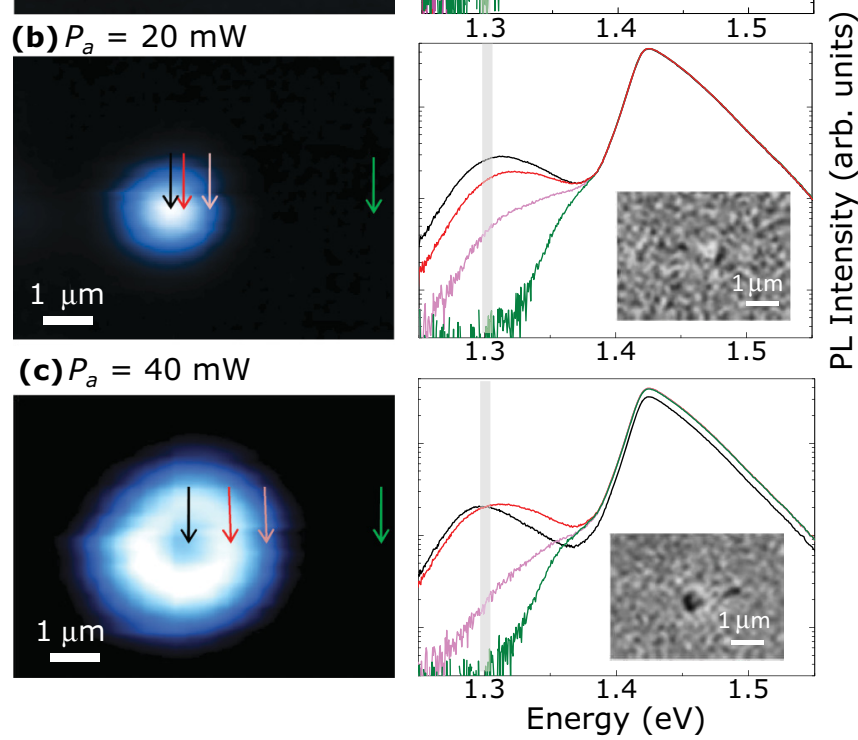

FIG. 1. (Color online) $\mu \mathrm{PL}$ maps at $T=300 \mathrm{~K}$ of an hydrogenated $\mathrm{Ga}(\mathrm{AsN}) \mathrm{QW}$ sample (H2) following exposure for a time $t_{a}=15 \mathrm{~s}$ to a focused laser beam with $\lambda=515 \mathrm{~nm}(h v=2.41 \mathrm{eV})$ and (a) $P_{a}=10 \mathrm{~mW}$, (b) $20 \mathrm{~mW}$, and (c) $40 \mathrm{~mW}$. The $\mu \mathrm{PL}$ spectra at positions $\mathrm{A}, \mathrm{B}, \mathrm{C}$, and $\mathrm{D}$ are shown at each power on the right. The $\mu \mathrm{PL}$ maps are obtained by plotting the $\mu \mathrm{PL}$ intensity around $E=1.30 \mathrm{eV}$ corresponding to the peak energy of the QW emission in the as-grown sample (see gray area in the $\mu \mathrm{PL}$ spectra) and were acquired with $P=1.2 \mathrm{~mW}$ and $\lambda=633 \mathrm{~nm}(h v=1.96 \mathrm{eV})$. Insets in (b) and (c) are $1 \mathrm{kV}$ SEM images of the laser-annealed spots.

(FWHM) of $0.95 \mu \mathrm{m}$ that corresponds closely to the laser spot diameter. With increasing laser power the light emitting areas increase in size showing spotlike [Figs. 1(a) and 1(b)] or ringlike [Fig. 1(c)] patterns, whose origin will be discussed later.

The incorporation of hydrogen in $\mathrm{Ga}(\mathrm{AsN})$ leads to the formation of several complexes. ${ }^{18-20}$ Dihydrogen complexes are responsible for the neutralization of the electronic activity of nitrogen, which causes the band gap and lattice constant of the $\mathrm{N}$-free $\mathrm{GaAs}$ to be recovered. In our experiment, this neutralization effect is reversed locally using a focused laser beam, which acts to dissociate the complex, thus restoring the electronic activity of nitrogen. ${ }^{11}$ We use the peak energy of the QW PL emission at different positions in each $\mu \mathrm{PL}$ map [Figs. 1(a)-1(c)] to probe the spatial distribution of active $\mathrm{N}$ and hence the percentage variation in the concentration of dihydrogen N-H complexes, $\Delta n_{\mathrm{N}-\mathrm{H}}$. Our estimate of $\Delta n_{\mathrm{N}-\mathrm{H}}$ uses a two-level band-anticrossing model with an interaction parameter $V_{\mathrm{N}}=2.7 \mathrm{eV}$ and a $\mathrm{N}$ level located at $0.23 \mathrm{eV}$ above the conduction band minimum of GaAs at $T=300 \mathrm{~K} \cdot{ }^{15}$ The values of $\Delta n_{\mathrm{N}-\mathrm{H}}$ at various laser powers and positions $X$ in the QW plane are plotted in Fig. 2(a). It can be 
clearly seen that with increasing $P_{a}$ : (i) the minimum in the $\Delta n_{\mathrm{N}-\mathrm{H}}$ profile becomes deeper; (ii) all $\mathrm{N}-\mathrm{H}$ complexes in the $\mathrm{Ga}(\mathrm{AsN}) \mathrm{QW}$ can be dissociated at laser powers $P_{a} \sim 40 \mathrm{~mW}$ corresponding to $\left|\Delta n_{\mathrm{N}-\mathrm{H}}\right| \sim 100 \%$; and (iii) the FWHM $d_{\mathrm{N}-\mathrm{H}}$ of the $\Delta n_{\mathrm{N}-\mathrm{H}}(X)$ curve increases above a minimum value corresponding to the diffraction-limited size of the laser spot diameter.

To examine the contribution of laser-induced heating of the crystal lattice on the ionization of the N-H complex and corresponding $\Delta n_{\mathrm{N}-\mathrm{H}}$ profiles, we first model the spatial gradient of the lattice temperature $T_{a}$ induced by the focused laser beam on the sample surface. Figure 2(b) shows the $X$ dependence of $T_{a}$ at various laser powers calculated by using the $T$-dependent thermal conductivity ${ }^{21}$ and reflection/absorption coefficients of GaAs at $\lambda=515 \mathrm{~nm}(h v=2.41 \mathrm{eV})$ and $T=300 \mathrm{~K}^{22}$ For $P_{a}<40 \mathrm{~mW}$, the calculated temperatures are well below those required $\left(T_{a}>250{ }^{\circ} \mathrm{C}\right)$ for thermal dissociation of $\mathrm{N}-\mathrm{H}$ complexes and outgassing of $\mathrm{H}$ from the sample, as measured in our high-temperature furnace experiments with $T$ up to $450{ }^{\circ} \mathrm{C}$ and as also found in the literature. ${ }^{18,19}$ Thus, under these laser exposure conditions, laser heating cannot be responsible for the ionization of the $\mathrm{N}-\mathrm{H}$ bonds. This is also supported by our laser writing experiments at liquid-helium temperatures $(T=4.2 \mathrm{~K})$ and laser exposure conditions $\left(P_{a}<\right.$ $40 \mathrm{~mW}$ ) that correspond to calculated temperatures $T_{a}<25 \mathrm{~K}$ (not shown).

In contrast, we find that for room temperature laser exposure at $P_{a} \geqslant 40 \mathrm{~mW}$, the temperature increases very steeply in the center of the laser spot. This thermal runaway effect is caused by the low thermal conductivity of GaAs at high temperatures. Note that the temperature can rise up to values that can exceed those required for $\mathrm{H}$ outgassing $\left(T_{a}>250^{\circ} \mathrm{C}\right)$, As desorption $\left(T_{a}>400{ }^{\circ} \mathrm{C}\right),{ }^{23}$ and melting of $\operatorname{GaAs}\left(T_{a}>1200^{\circ} \mathrm{C}\right)$. Thus, at high laser powers $\left(P_{a} \geqslant 40 \mathrm{~mW}\right)$, the high temperatures $\left(T_{a} \geqslant 250^{\circ} \mathrm{C}\right)$ induced around the laser spot can cause thermal dissociation of the complex, diffusion and outgassing of $\mathrm{H}$. This results in a broadening of the $\Delta n_{\mathrm{N}-\mathrm{H}}$ profile to full widths at half maximum, $d_{\mathrm{N}-\mathrm{H}}$, that exceed the diffraction-limited size of the laser beam [Fig. 2(c)], and in irreversible damage of the sample surface [see darker spot in the SEM and $\mu \mathrm{PL}$ maps of Fig. 1(c)]. The measured values of $d_{\text {crater }}$, i.e., the diameter of the laser-induced surface damage revealed by SEM, increases with increasing $P_{a}$ in qualitative agreement with the estimated width of the laser-annealed region at temperatures $T_{a}>400^{\circ} \mathrm{C}$ [see Fig. 2(c)]. No topographic changes can instead be revealed in samples laser annealed at lower powers $\left(P_{a}<40 \mathrm{~mW}\right)$. For these laser exposure conditions, AFM images show an atomically flat surface, whereas the SE image reveals only a weak contrast variation, which we attribute to the laser-induced change of the $\mathrm{N}-\mathrm{H}$ complex concentration. ${ }^{24}$

Various processes can be envisaged to explain the laserassisted dissociation of the N-H complex without invoking thermal effects: ${ }^{8-10}$ the photogenerated electron-hole pairs recombine nonradiatively and release their energy to the complex; the complex absorbs directly the incoming photon and breaks up if the photon energy is equal or larger than its dissociation energy; or multiple vibrational excitation can act to break up the $\mathrm{N}-\mathrm{H}$ bond, as observed for other H-related (a)

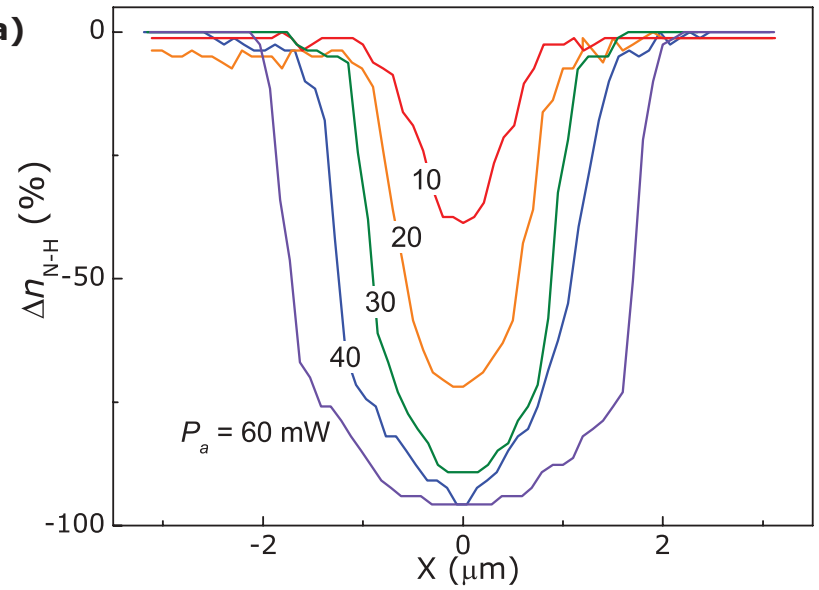

(b)

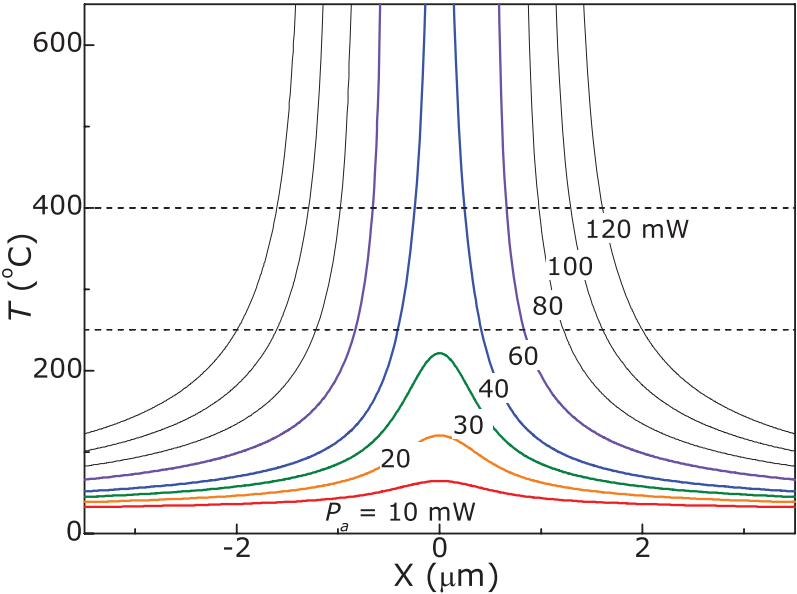

(c)

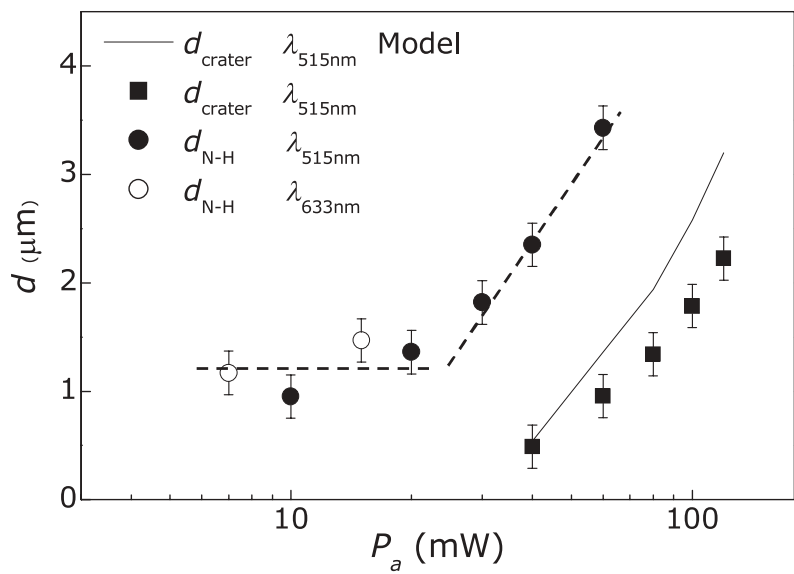

FIG. 2. (Color online) (a) $X$ dependence of the percentage variation in the concentration of dihydrogen N-H complexes $\Delta n_{\mathrm{N}-\mathrm{H}}$ at various $P_{a}\left(\lambda=515 \mathrm{~nm}, h v=2.41 \mathrm{eV}, t_{a}=15 \mathrm{~s}\right)$ in the hydrogenated $\mathrm{Ga}(\mathrm{AsN}) \mathrm{QW}$ (sample H2). (b) Calculated $T_{a}(X)$ curves at various $P_{a}\left(\lambda=515 \mathrm{~nm}, h v=2.41 \mathrm{eV}, t_{a}=15 \mathrm{~s}\right.$, beam waist $0.4 \mu \mathrm{m})$. Horizontal dashed lines highlight temperatures required for $\mathrm{H}$ outgassing $\left(T_{a}>250{ }^{\circ} \mathrm{C}\right)$ and As desorption $\left(T_{a}>\right.$ $400{ }^{\circ} \mathrm{C}$ ). (c) Dependence of the full widths at half maximum of the $\Delta n_{\mathrm{N}-\mathrm{H}}$ profile $d_{\mathrm{N}-\mathrm{H}}$ on $P_{a}$ at $\lambda=515 \mathrm{~nm}(h v=2.41 \mathrm{eV})$ and $633 \mathrm{~nm}(h v=1.96 \mathrm{eV})$. The dashed line is a guide to the eye. The squares and line describe the $P_{a}$ dependence of $d_{\text {crater }}$ (the diameter of the damaged surface area of the sample) as derived from the SEM study and our estimate based on the As-desorption model, respectively. 


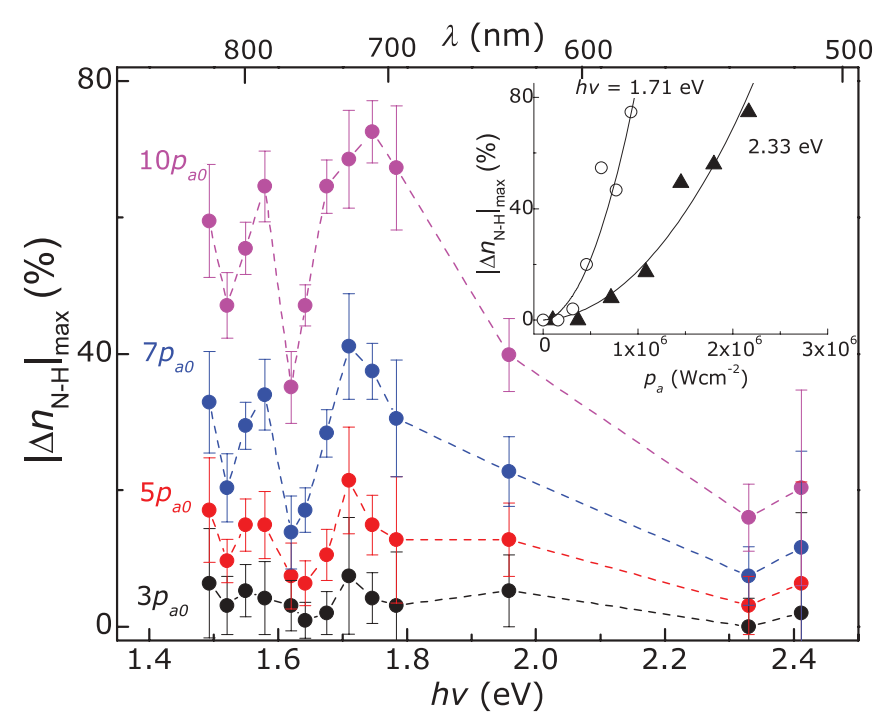

FIG. 3. (Color online) Dependence of the value of the $\left|\Delta n_{\mathrm{N}-\mathrm{H}}(X)\right|$ profile at the center $(X=0)$ of the laser spot, $\left|\Delta n_{\mathrm{N}-\mathrm{H}}\right|_{\max }$, on the photon energy $h v$ and laser wavelength $\lambda$ at various power densities $p_{a}$ and $t_{a}=20 \mathrm{~s}\left(p_{a 0}=10^{5} \mathrm{Wcm}^{-2}\right)$. The inset shows the dependence of $\left|\Delta n_{\mathrm{N}-\mathrm{H}}\right|_{\max }$ on $p_{a}$ at $h v=1.71$ and $2.33 \mathrm{eV}(\lambda=725$ and $532 \mathrm{~nm})$. Continuous lines are quadratic fits to the data.

complexes in diamond ${ }^{9}$ and silicon ${ }^{10}$ in electron-beam and electron tunneling experiments. We examine the plausibility of these mechanisms by studying the dependence of $\left|\Delta n_{\mathrm{N}-\mathrm{H}}\right|$ on the laser power density $p_{a}$ and photon wavelength $\lambda$ (Fig. 3). Here the photon wavelength is tuned over a range of energies $h v=1.49-2.41 \mathrm{eV}(\lambda=830-515 \mathrm{~nm})$, below and above the dissociation energy $E_{\mathrm{A}}=1.77-1.89 \mathrm{eV}(\lambda=700-656 \mathrm{~nm})$ of the $\mathrm{N}-\mathrm{H}$ complexes as derived from thermal annealing studies of $\mathrm{Ga}(\mathrm{AsN}){ }^{19}$ For each $\lambda$ and $p_{a}$, we mapped the $\Delta n_{\mathrm{N}-\mathrm{H}}$ profile and estimated the value of $\Delta n_{\mathrm{N}-\mathrm{H}}$ at the center of each laser spot, $\left|\Delta n_{\mathrm{N}-\mathrm{H}}\right|_{\max }$.

From Fig. 3, it can be seen that at each power the value of $\left|\Delta n_{\mathrm{N}-\mathrm{H}}\right|_{\max }$ tends to peak at $h v=1.76 \mathrm{eV}(\lambda \sim 700 \mathrm{~nm})$, close to the reported values of $E_{\mathrm{A}} \cdot{ }^{19}$ Photoluminescence excitation measurements of both hydrogenated and untreated samples exclude the presence of specific resonances in the density of states of the crystal at the energy mentioned above. Thus the data in Fig. 3 suggest a resonant photon absorption by the N-H complex itself accompanied by its dissociation. Perhaps surprisingly, the phenomenon is also observed under laser photon energies that are significantly smaller than $E_{\mathrm{A}}$. To explain this result, we envisage that the dissociation can be assisted by phonons and that the photogenerated electron-hole pairs can act to screen the N-H chemical bond, effectively reducing the dissociation energy of the complex. Similar mechanisms were invoked to explain the light-enhanced $\mathrm{H}$ diffusion in amorphous $\mathrm{Si}^{8}$ and the photoinduced reactivation of neutralized donors in Si-doped $\mathrm{GaAs},{ }^{25}$ although a photon-induced dissociation of H-related complexes has never been considered before. The data in Fig. 3 also reveal a weak enhancement of $\left|\Delta n_{\mathrm{N}-\mathrm{H}}\right|_{\max }$ at photon excitation energies of $h v=1.5-1.6 \mathrm{eV}$, lower than the main peak. This feature suggests a lower energy bound for the dissociation of the dihydrogen complex by the formation of a closely related complex state with a lower activation energy $E_{A}^{*}=E_{A}-0.2 \mathrm{eV}^{20}$

Plots of $\left|\Delta n_{\mathrm{N}-\mathrm{H}}\right|$ versus the laser power density indicate that at all laser wavelengths, the values of $\left|\Delta n_{\mathrm{N}-\mathrm{H}}\right|$ exhibit a nonlinear dependence on $p_{a}$ (see quadratic fit to the data in the inset of Fig. 3). This behavior is consistent with a multiphoton absorption process, i.e., two photons are required to break up the dihydrogen $\mathrm{N}-\mathrm{H}$ complex to fully restore the electronic activity of the $\mathrm{N}$ atoms. The excitation of an individual $\mathrm{N}-\mathrm{H}$ bond would be sufficient to restore the band-gap energy of $\mathrm{Ga}$ (AsN). However, a single N-H bond is less stable than a dihydrogen $\mathrm{N}-\mathrm{H}$ complex ${ }^{26}$ and although we cannot exclude that a single N-H bond may be left behind following the laser annealing, our photoluminescence studies do not provide any direct evidence for this process. The measured dependence of $\left|\Delta n_{\mathrm{N}-\mathrm{H}}\right|$ on $p_{a}$ is also likely to be affected by the nonlinear dependence of the dissociation rate on the laser power due to the contribution of vibrational excitations. ${ }^{9}$ Theoretical models and further experimental studies (e.g., Raman studies) are required to assess these peculiar aspects of the $\mathrm{N}-\mathrm{H}$ complex photon-assisted dissociation in III-N-Vs.

The dominant photonic nature of the laser-induced dissociation of the N-H complex at low laser powers implies that the size of the laser-annealed spot is mainly limited by light diffraction, i.e., it is set by the laser spot diameter $d=1.22 \lambda / \mathrm{NA}$, where NA $=0.8(0.9)$ is the numerical aperture of our $\times 100$ objectives. For $\lambda=515(h v=2.41 \mathrm{eV})$ and $633 \mathrm{~nm}(h v=1.96 \mathrm{eV})$, this gives $d \sim 0.7-0.8$ and $0.9-1 \mu \mathrm{m}$, respectively, in good agreement with the measured $\Delta n_{\mathrm{N}-\mathrm{H}}(X)$

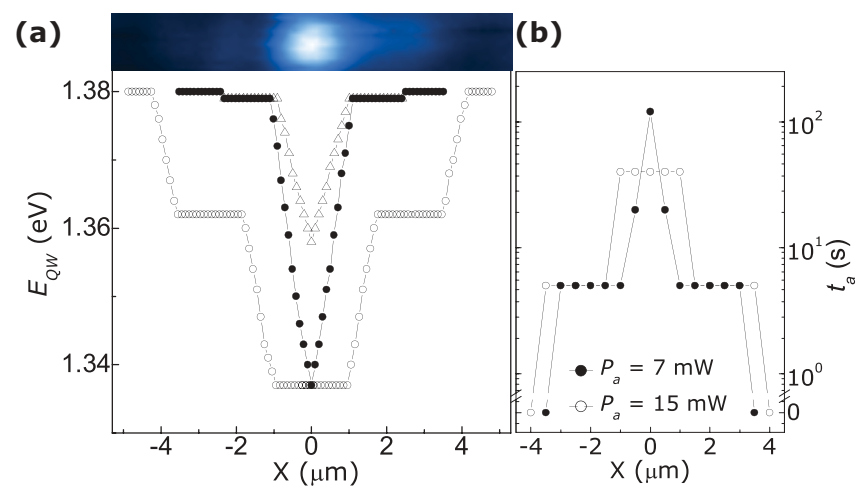

(c)
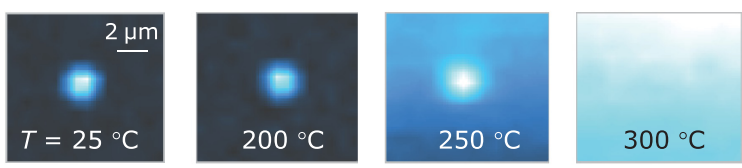

FIG. 4. (Color online) (a) Profiles of the QW PL peak energy $E_{\mathrm{QW}}$ created by laser writing in the hydrogenated Ga(AsN) QW (sample $\mathrm{H} 2$ ). The top figure is the $\mu \mathrm{PL}$ map corresponding to the triangular potential well (see triangles in the main figure). (b) Annealing times and powers used for different profiles (triangular profile, full symbols; steplike profile, empty symbols). (c) Light emitting spots in sample $\mathrm{H} 1$ created by laser writing $\left(P_{a}=20 \mathrm{~mW}, \lambda=515 \mathrm{~nm}\right.$, $h v=2.41 \mathrm{eV}$, and $t_{a}=15 \mathrm{~s}$ ) and mapped following an annealing in a high-temperature furnace at $T=200^{\circ} \mathrm{C}, 250^{\circ} \mathrm{C}$, and $300^{\circ} \mathrm{C}$ for $1 \mathrm{~h}$. All $\mu \mathrm{PL}$ maps are obtained by integrating the PL intensity around the peak energy of the QW emission in the as-grown sample $(E=1.30 \mathrm{eV})$ 


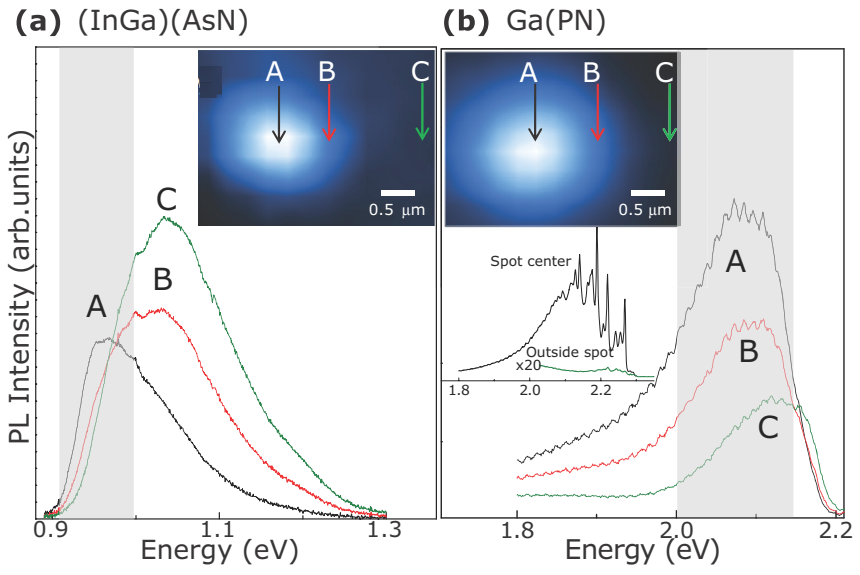

FIG. 5. (Color online) (a) $\mu \mathrm{PL}$ map and $\mu \mathrm{PL}$ spectra at positions $\mathrm{A}, \mathrm{B}$, and $\mathrm{C}$ of a hydrogenated (InGa)(AsN) QW following laser writing at $T=300 \mathrm{~K}\left(t_{a}=20 \mathrm{~s}, P_{a}=7 \mathrm{~mW}, \lambda=532 \mathrm{~nm}, h v=2.33 \mathrm{eV}\right)$. The $\mu \mathrm{PL}$ maps are obtained by plotting the $\mu \mathrm{PL}$ intensity around $E=0.9-1 \mathrm{eV}$, corresponding to the energy range of the QW emission in the as-grown sample (see shaded area) and were acquired at low laser powers $(\lambda=633 \mathrm{~nm}, h v=1.96 \mathrm{eV}, P=0.1 \mathrm{~mW}, T=300 \mathrm{~K})$. (b) $\mu \mathrm{PL}$ map and $\mu \mathrm{PL}$ spectra at positions $\mathrm{A}, \mathrm{B}$, and $\mathrm{C}$ of a hydrogenated $\mathrm{Ga}(\mathrm{PN}) \mathrm{MQW}$ following laser writing at $T=300 \mathrm{~K}$ $\left(t_{a}=20 \mathrm{~s}, P_{a}=22 \mathrm{~mW}, \lambda=532 \mathrm{~nm}, h v=2.33 \mathrm{eV}\right)$. The $\mu \mathrm{PL}$ maps are obtained by plotting the $\mu \mathrm{PL}$ intensity around $E=2.0-2.15 \mathrm{eV}$ corresponding to the energy range of the MQW emission in the as-grown sample (see shaded area) and were acquired at low laser powers $(\lambda=532 \mathrm{~nm}, h v=2.33 \mathrm{eV}, P=0.9 \mathrm{~mW}, T=10 \mathrm{~K})$. The inset shows the $\mu \mathrm{PL}$ spectra at $T=20 \mathrm{~K}$ of the $\mathrm{Ga}(\mathrm{PN})$ bulk sample inside and outside a typical laser spot created with $\lambda=532 \mathrm{~nm}(h v=$ $2.33 \mathrm{eV}$ )

profiles. Thus direct laser writing can provide a versatile tool for in situ in-plane band-gap engineering. Potential profiles of different shapes can be created and controlled with submicron spatial resolution and high energy accuracy ( $\sim 1 \mathrm{meV}$ ) by adjusting the laser power and/or exposure time. Figure 4(a) illustrates examples of spatial modulation of the $\mathrm{Ga}(\mathrm{AsN}) \mathrm{QW}$ PL peak energy $E_{\mathrm{QW}}$ obtained by scanning a laser beam over the surface of sample H2: Triangular and steplike in-plane potential wells of given energy depth and spatial width are patterned in the $X Y$ plane of the $\mathrm{QW}$. The specific annealing conditions used to create two of the potential profiles are illustrated in Fig. 4(b). Thermal annealing studies in a high-temperature furnace show that the light emitting areas created by laser are stable up to temperatures $T \sim 200^{\circ} \mathrm{C}$ and that they can be erased at $T>250{ }^{\circ} \mathrm{C}$ due to the thermal dissociation of the $\mathrm{N}-\mathrm{H}$ complexes and $\mathrm{H}$ outgassing from the whole sample [see Fig. 4(c)]. Thus the local dehydrogenation effect achieved by laser writing corresponds to temperatures that are considerably smaller than those required for thermal dissociation $\left(T>200^{\circ} \mathrm{C}\right)$; this effect is erasable and the electronic properties of the as-grown (nonhydrogenated) QW can be fully restored by a mildtemperature thermal annealing. The same sample can be rehydrogenated making any nanoscale in-plane band-gap profile rewritable.

Our experiments in the hydrogenated ( $\mathrm{InGa})(\mathrm{AsN})$ and $\mathrm{Ga}(\mathrm{PN})$ samples reveal effects that are qualitatively similar to those described for $\mathrm{Ga}(\mathrm{AsN})$. Figures 5(a) and 5(b) show the $\mu \mathrm{PL}$ spectra and $\mu \mathrm{PL}$ maps for the $(\mathrm{InGa})(\mathrm{AsN})$ and $\mathrm{Ga}(\mathrm{PN})$ QWs following laser exposure to a focused laser beam with $\lambda=532 \mathrm{~nm}(h v=2.33 \mathrm{eV})$. It can be seen that the PL emission is peaked at lower energies in proximity of the spot center. The increase of PL intensity induced by laser writing in the $\mathrm{Ga}(\mathrm{PN}) \mathrm{MQW}$ [Fig. 5(b)] becomes more pronounced in the bulk Ga(PN) sample [inset of Fig. 5(b)]. The hydrogenated $\mathrm{Ga}(\mathrm{PN})$ bulk sample contains only a small concentration of $\mathrm{N}$ $([\mathrm{N}]=0.15 \%)$ and the $\mathrm{H}$ atoms act to fully neutralize the electronic activity of $\mathrm{N}$, thus leading to a weak PL emission similar to that of $\mathrm{Ga}(\mathrm{PN})$ in the limit of very diluted $\mathrm{N}$ concentration $([\mathrm{N}]<0.01 \%)$, where the recombination of excitons bound to single $\mathrm{N}$ atoms dominates the spectrum [see inset of Fig. 5(b)]. Following the laser exposure, strong narrow emission lines can be clearly seen in the spectrum and are associated with carrier recombination from N-related complexes. $^{27,28}$ Importantly, the $\mathrm{N}-\mathrm{H}$ dissociation can be activated in our samples for laser photon wavelengths above $(\lambda=532 \mathrm{~nm} ; h v=2.33 \mathrm{eV})$ and below $(\lambda=633 \mathrm{~nm}$; $h v=1.96 \mathrm{eV})$ the band-gap absorption wavelength of $\mathrm{GaP}$ $(\lambda=550 \mathrm{~nm} ; h v=2.25 \mathrm{eV})$. This is in line with a direct photon absorption by the N-H complex, rather than a thermal effect.

\section{CONCLUSIONS}

In conclusion, we have shown that the dissociation of dihydrogen $\mathrm{N}-\mathrm{H}$ complexes in hydrogenated III-N-Vs can be achieved by low laser powers, corresponding to local temperatures much smaller than those required for thermal dissociation $\left(>200^{\circ} \mathrm{C}\right)$. The photon absorption by the $\mathrm{N}-\mathrm{H}$ complex mediated by photogenerated carriers provides a mechanism for band-gap profiling: profiles of different shapes can be patterned with submicron spatial resolution and high energy accuracy in the growth plane of the III-N-Vs; the profiles are erasable and the sample can be rehydrogenated making any nanoscale in-plane band-gap profile rewritable. The possibility of in-plane profiling of the band-gap energy offers flexibility in the control and exploitation of the electronic properties of a semiconductor without the implementation of lithographic/etching techniques. The versatility of hydrogen makes this direct laser writing approach of general interest and relevant to the development of fast and easy fabrication approaches to nanotechnologies. Nonthermal (photonic) laser writing may offer an alternative promising route to nondestructive band-gap profiling of temperature-sensitive material systems. Also, it advances prospects of using hydrogen in III-N-Vs in future nanotechnologies.

\section{ACKNOWLEDGMENTS}

This work was supported by The Royal Society, the University of Nottingham, the EU (under Grant Agreement No. PIEF-GA-2010-272612), and the COST Action MP0805. We acknowledge C. Tu, K. Volz, W. Stolz, A. Forchel, and M. Hopkinson for growing the (InGa)(AsN) and $\mathrm{Ga}(\mathrm{PN})$ samples. 
*Corresponding author: amalia.patane@ nottingham.ac.uk

${ }^{1}$ J. Chevallier and M. Aucouturier, Annu. Rev. Mater. Sci. 18, 219 (1988).

${ }^{2}$ C. G. Van de Walle and J. Neugebauer, Annu. Rev. Mater. Res. 36, 179 (2006).

${ }^{3}$ C. G. Van de Walle and J. Neugebauer, Nature (London) 423, 626 (2003).

${ }^{4}$ C. G. Van de Walle and B. Tuttle, IEEE Trans. Electron Devices 47, 1779 (2000).

${ }^{5}$ D. C. Elias, R. R. Nair, T. M. G. Mohiuddin, S. V. Morozov, P. Blake, M. P. Halsall, A. C. Ferrari, D. W. Boukhvalov, M. I. Katsnelson, A. K. Geim, and K. S. Novoselov, Science 323, 610 (2009).

${ }^{6}$ E. A. Davis, S. F. J. Cox, R. L. Lichti, and C. G. Van de Walle, Appl. Phys. Lett. 82, 592 (2003).

${ }^{7}$ R. Trotta, A. Polimeni, F. Martelli, G. Pettinari, M. Capizzi, L. Felisari, S. Rubini, M. Francardi, A. Gerardino, P. C. M. Christianen, and J. C. Maan, Adv. Mater. 23, 2706 (2011).

${ }^{8}$ P. V. Santos, N. M. Johnson, and R. A. Street, Phys. Rev. Lett. 67, 2686 (1991).

${ }^{9}$ N. Habka, J. Chevallier, and J. Barjon, Phys. Rev. B 81, 045207 (2010).

${ }^{10}$ T.-C. Shen, C. Wang, G. C. Abeln, J. R. Tucker, J. W. Lyding, Ph. Avouris, and R. E. Walkup, Science 268, 1590 (1995).

${ }^{11}$ N. Balakrishnan, A. Patanè, O. Makarovsky, A. Polimeni, M. Capizzi, F. Martelli, and S. Rubini, Appl. Phys. Lett. 99, 021105 (2011).

${ }^{12}$ R. Carron, D. Fekete, P. Gallo, B. Dwir, A. Rudra, M. Felici, B. Bartova, M. Cantoni, and E. Kapon, Appl. Phys. Lett. 99, 101107 (2011).

${ }^{13}$ N. Lopez, L. A. Reichertz, K. M. Yu, K. Campman, and W. Walukiewicz, Phys. Rev. Lett. 106, 028701 (2011).

${ }^{14} \mathrm{M}$. Henini, Dilute Nitride Semiconductors (Elsevier, Amsterdam, 2005).
${ }^{15}$ W. Shan, W. Walukiewicz, J. W. Ager, E. E. Haller, J. F. Geisz, D. J. Friedman, J. M. Olson, and S. R. Kurtz, Phys. Rev. Lett. 82, 1221 (1999).

${ }^{16}$ R. Trotta, D. Giubertoni, A. Polimeni, M. Bersani, M. Capizzi, F. Martelli, S. Rubini, G. Bisognin, and M. Berti, Phys. Rev. B 80, 195206 (2009).

${ }^{17}$ M. Berti, G. Bisognin, D. De Salvador, E. Napolitani, S. Vangelista, A. Polimeni, M. Capizzi, F. Boscherini, G. Ciatto, S. Rubini, F. Martelli, and A. Franciosi, Phys. Rev. B 76, 205323 (2007).

${ }^{18}$ G. Ciatto, F. Boscherini, A. A. Bonapasta, F. Filippone, A. Polimeni, and M. Capizzi, Phys. Rev. B 71, 201301(R) (2005).

${ }^{19}$ G. Bisognin, D. De Salvador, E. Napolitani, M. Berti, A. Polimeni, M. Capizzi, S. Rubini, F. Martelli, and A. Franciosi, J. Appl. Crystallogr. 78, 366 (2008).

${ }^{20}$ A. Bonapasta, F. Filippone, and G. Mattioli, Phys. Rev. Lett. 98, 206403 (2007).

${ }^{21}$ M. Lax, J. Appl. Phys. 48, 3919 (1977).

${ }^{22}$ J. S. Blakemore, J. Appl. Phys. 53, R123 (1982).

${ }^{23}$ N. Kobayashi and Y. Kobayashi, Jpn. J. Appl. Phys., Part 2 30, L1699 (1991).

${ }^{24}$ L. Felisari, V. Grillo, F. Martelli, R. Trotta, A. Polimeni, M. Capizzi, F. Jabeen, and L. Mariucci, Appl. Phys. Lett. 93, 102116 (2008).

${ }^{25}$ D. Loridant-Bernard, S. Meziere, M. Constant, N. Dupuy, S. Sombret, and J. Chevalier, Appl. Phys. Lett. 73, 644 (1998).

${ }^{26}$ A. Janotti, S. B. Zhang, Su-Huai Wei, and C. G. Van de Walle, Phys. Rev. Lett. 89, 086403 (2002).

${ }^{27}$ A. Polimeni, M. Bissiri, M. Felici, M. Capizzi, I. A. Buyanova, W. M. Chen, H. P. Xin, and C. W. Tu, Phys. Rev. B 67, 201303R (2003).

${ }^{28}$ H. P. Xin, C. W. Tu, Y. Zhang, and A. Mascarenhas, Appl. Phys. Lett. 76, 1267 (2000). 